\title{
Giant Prolactinoma and Concomitant Internal Carotid Artery Pseudo-Aneurysm: All Stages of the Decision-Making Process
}

\author{
A Giorgianni ${ }^{1}$, P Veiceschi ${ }^{2 *}$, G Agresta ${ }^{2}, S$ Balbi ${ }^{3}$ and D Locatelli ${ }^{2}$ \\ ${ }^{1}$ Department of Neuroradiology, University of Insubria, Varese, Italy \\ ${ }^{2}$ Department of Neurosurgery, University of Insubria, Varese, Italy \\ ${ }^{3}$ Department of Biotechnology and Life Sciences, Unit of Neurosurgery, University of Insubria, Varese, Italy
}

*Corresponding author: P Veiceschi, MD, Department of Neurosurgery, Ospedale di Circolo Fondazione Macchi, Via Guicciardini 9, 21100 Varese, Italy

\begin{abstract}
We report a case of right internal carotid artery pseudo-aneurysm, arising in the aspects of a giant macroadenoma in a 57-years-old man, presented with right severe temporal orbital headache and vomiting.

Computed tomography scan $(\mathrm{CT})$ revealed a destructive skull base mass extended from sellar region to clivus. Pre and post-gadolinium brain magnetic resonance (MRI) confirmed the lesion, characterized by an encasement of optic nerves and carotid siphons bilaterally, with a pseudo-aneurysm of cavernous segment of the right internal carotid artery (ICA).

The patient underwent endoscopic biopsy of the endonasal portion of the lesion with a subsequent diagnosis of prolactinoma. Endovascular therapy using Derivo ${ }^{\circledR} 5 \times 25 \mathrm{~mm}$, Acandis $^{\circledR}$ flow diversion embolization device was performed. After subsequent hormonal therapy with Cabergoline, a six months follow-up MRI and digital subtractions angiography (DSA) demonstrated reduction of both tumour and pseudo-aneurysm volume.
\end{abstract}

\section{Keywords}

Anterior pituitary, Neuroendocrinology, Prolactinoma, Pseudo-aneurysm, Decision making

\section{Introduction}

The coexistence of a cerebral aneurysm and a pituitary adenoma is common, and it is well described in the literature [1,2] with an incidence of $3.7 \%$ to $7.4 \%$ [3]. However, the coexistence of an aneurysm with sellar extension and pituitary adenoma is rare [4].
In this case, authors underline the requirement to plan the optimal timing to treat both lesions with an effective methodology and to avoid terrible complications like accidental rupture of the aneurysm during surgery.

\section{Case Presentation}

A 57-years-old man referred mild headache during the last two months with recent episode of acute severe right orbital headache and neck pain, occurred during Valsalva manoeuvre. At admission in our department, no cranial nerves or visual fields deficits were detected.

Non-contrast head CT revealed a large destructive central skull base mass with hyperdensity in his context. A further examination with pre-and post-gadolinium brain MRI, underlined the limits of the skull base mass centred in cellar region with erosion of sphenoid sinus and clivus. It was extended inferiorly to the magnum foramen and occipital condyles, laterally to the petrous apices, sigmoid sinus and jugular bulbs; superiorly occupying the chiasmatic cistern, encasing optic nerves and both internal carotid arteries (Figure 1). A large flow void, within the region of the right carotid siphon has been shown (Figure 2). Digital subtraction angiography (DSA) was performed that confirmed a pseudo-aneurysm of cavernous segment of right ICA with a bleb on the anterior side (Figure 1).

Patient underwent endoscopic biopsy of the endonasal portion of the lesion: Histopathologic findings were consistent with pituitary adenoma. Subsequent

\footnotetext{
Citation: Giorgianni A, Veiceschi P, Agresta G, Balbi S, Locatelli D (2018) Giant Prolactinoma and Concomitant Internal Carotid Artery Pseudo-Aneurysm: All Stages of the Decision-Making Process. Neurosurg Cases Rev 1:007.

Accepted: November 17, 2018; Published: November 19, 2018

Copyright: (c) 2018 Giorgianni A, et al. This is an open-access article distributed under the terms of the Creative Commons Attribution License, which permits unrestricted use, distribution, and reproduction in any medium, provided the original author and source are credited.
} 

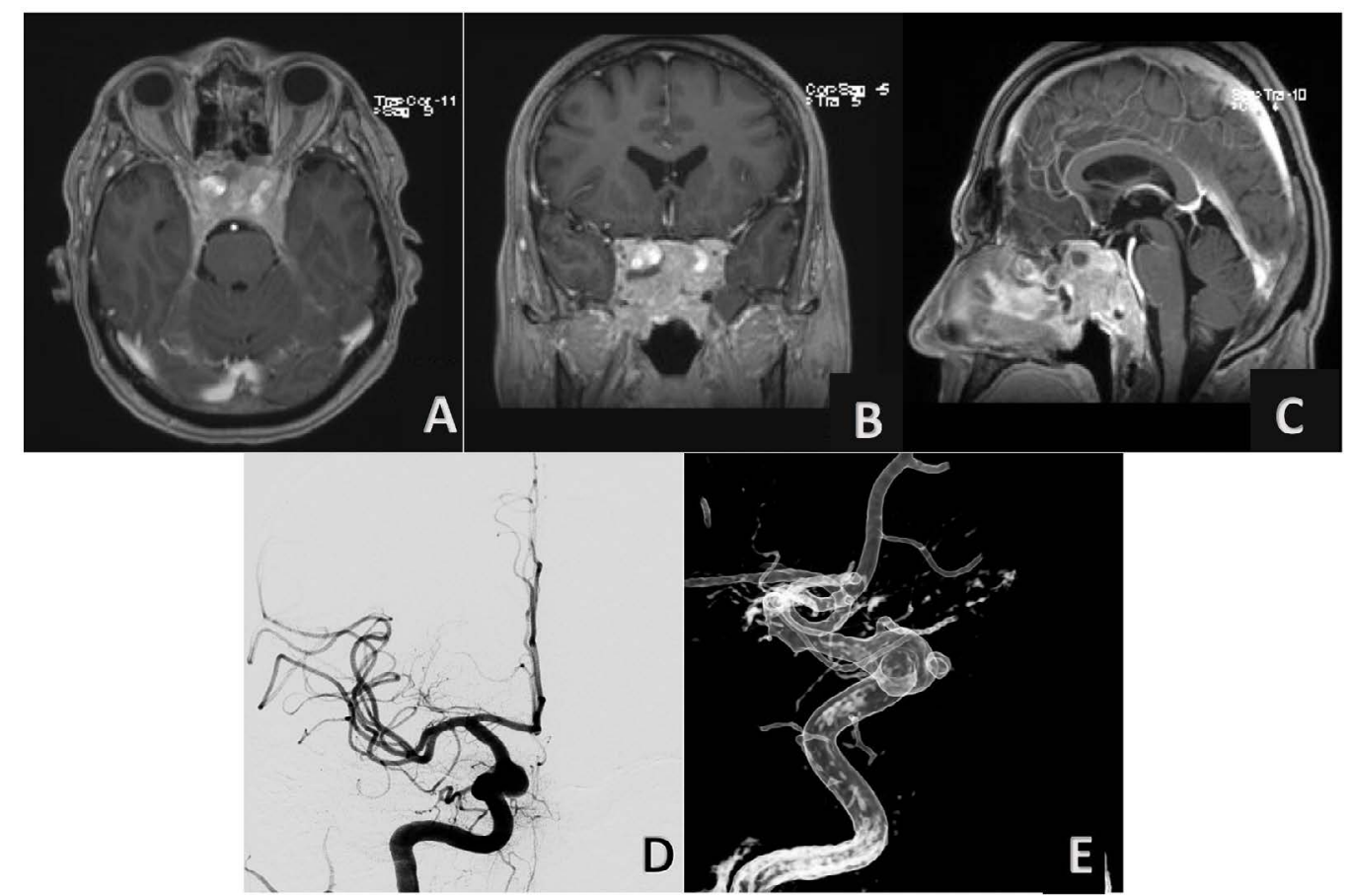

Figure 1: Contrast-enhanced T1-weighted magnetic resonance images in the axial A) Coronal; B) And sagittal planes; C) Demonstrate an intra and parasellar tumor; Four-vessels angiogram in anteroposterior; D) And three-dimensional lateral view; E) Reveals a large pseudo-aneurysm of cavernous segment of right ICA with bleb on anterior side.
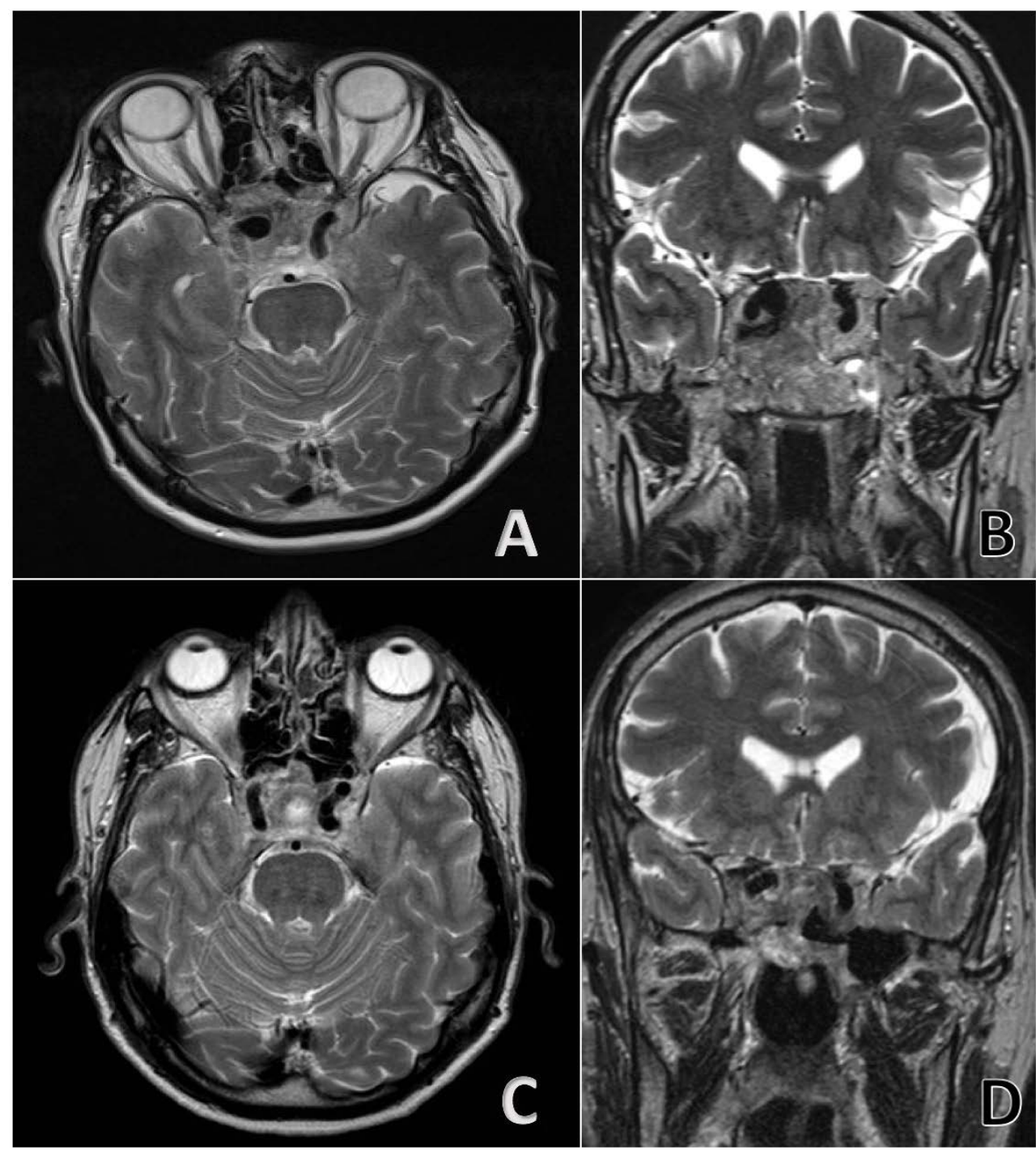

Figure 2: Non-Contrast T2-weighted MRI comparates pre-treatment (A,B) and post-treatment (C,D). 
biochemical testing revealed marked hyperprolactinemia (PRL $4700 \mathrm{ng} / \mathrm{mL}$ - normal range 2.1-17.7 ng/mL) with central hypogonadism, leading to a diagnosis of giant prolactinoma. Patient was treated with Aspirin and Clopidogrel for five days and then he underwent endovascular therapy with Derivo ${ }^{\circledR}\left(\right.$ Acandis $\left.^{\circledR}\right) 5 \times 25 \mathrm{~mm}$ flow diversion embolization device. Patient's headache resolved, and he was discharged in good condition four days after endovascular therapy. He was loaded with Aspirin and Clopidogrel for one month, followed by only Aspirin in the next three months.

After 15 days from endovascular treatment it was introduced medical therapy with dopamine receptor agonists to treat the prolactinoma. We choose Cabergoline, who has become the preferred first-line agent, and is associated with less frequent and less severe side effects and is easier to administer than bromocriptine, although it is little more expansive [5]. Medical therapy was started with low doses with progressive increase during the subsequent follow-up, in order to avoid a too fast volumetric reduction of the lesion and then the traction on the aneurysm sac, with possible aneurysm rupture, and for the intrinsic risk of the therapy to induce a pituitary apoplexy. However, no side effects occurred during treatment with Cabergoline, and patient serum prolactin level 3 months later was $4.7 \mathrm{ng} / \mathrm{ml}$.

Post-gadolinium MRI 6 months after starting medical treatment (Figure 3) showed significant tumor shrinkage with decompression of the optic apparatus. Furthermore DSA (Figure 3) demonstrated almost complete resolution of aneurysm. Patient continued to be in good health during follow-up, with no bleeding or other complications.

\section{Discussion}

The incidence of intracranial aneurysms arising in association with pituitary adenomas is greater than the incidence of aneurysms arising in the general population $5.4 \%$ [1], as well as the incidence of aneurysms coexistent with intracranial masses of non-pituitary origin [6]. However, the presence of an aneurysm inside a pituitary adenoma is rare [4]. In these cases, most aneurysms are asymptomatic and discovered accidentally during radiological investigations.

The association between these two phenomena is not clear. The coexistence of the aneurysm in close proximity to the adenoma may be due to various reasons, including the mechanical effect of adenoma on the vessels [7]. Mangiardi, et al. [8] described vascular infiltration as a possible cause of the formation of a pseudo aneurysm. In some cases of secreting adenoma GH, the production of this hormone was also considered to cause accelerated atherosclerosis and consequently degenerative changes on the vessel walls [9].

The optimal management of co-existing pseudo-aneurysm with pituitary adenoma is controversial and depends on the size of aneurysm, size and functional status of adenoma, associated apoplexy manifestation, eventually visual field deficits [4]. In absence of complications such as apoplexy, the treatment of the pseudo-aneurysm represents the priority before attempting surgical or medical intervention on pituitary adenoma. In this particular case we have faced two main issues: The risk of bleeding of a pseudo-aneurysm related to cytoreduction effect of Cabergoline and also possible pituitary apoplexy secondary to Cabergoline and concomitant double antiplatelet therapy [10-12].

It has been tried to optimize the timing, allowing a secure and effective treatment of both lesions. On account of the risk of rupture of aneurysm caused by traction on the wall, for volumetric regression of the adenoma after medical therapy [13], at first it was made safe the aneurysm, before starting any treatment kind on the adenoma.

The risk of rupture of the aneurysm connected with recent signs of lesion bleeding, led us besides to give priority to the treatment of the aneurysm itself and to
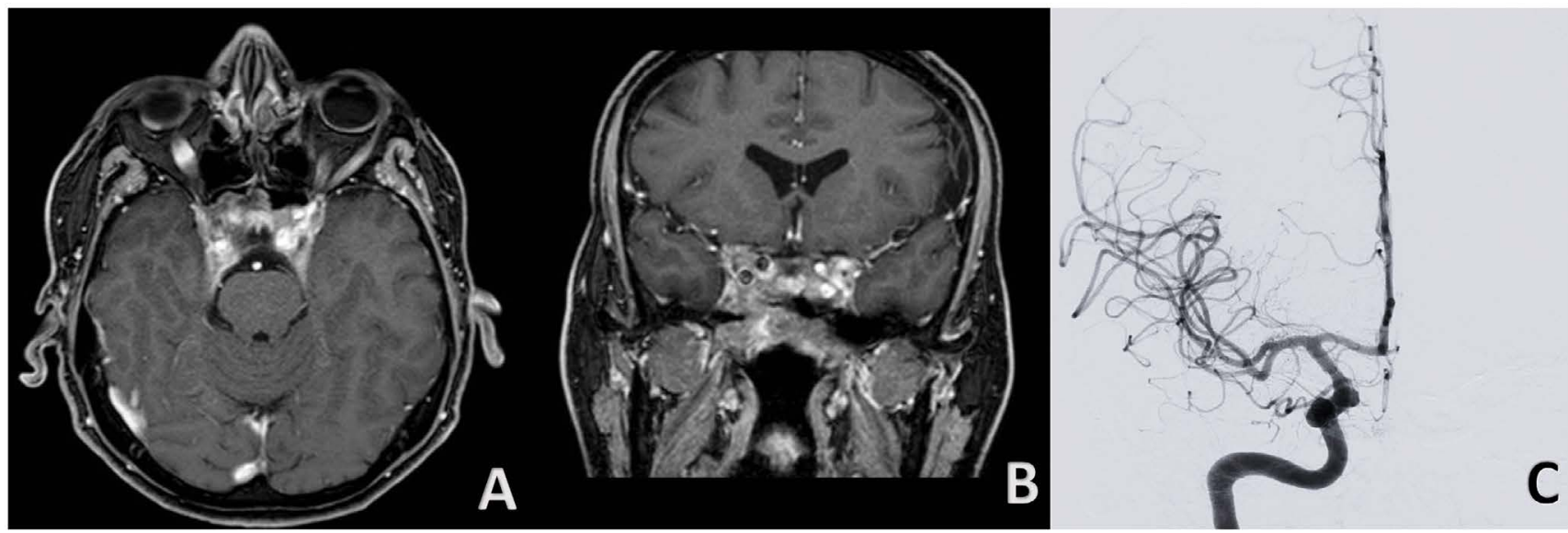

Figure 3: Contrast-enhanced T1-weighted magnetic resonance images in the axial A) And coronal; B) After treatment demonstrate considerable reduction of prolactinoma size; Four-vessels angiogram in anteroposterior view; C) Reveals almost complete recovery of pseudoaneurysm. 
avoid therefore exposing it to a breaking risk together with a medical advanced therapy. The aneurysm has been treated through endovascular methodology, a better indication in terms of outcome as regards microsurgical approaches-considered areas such as the cavernous segment or the paraclinoidal tract of the internal carotid [14]; thus, after two weeks it was started the low dose therapy with Cabergoline for the giant prolactinoma that has been progressively increased.

The patient has been therefore monitored with a strict follow-up and after about six months from the procedure, it has been noticed an excellent result on both lesions (Figure 2).

\section{Conclusions}

Unawareness about coexisting pseudo aneurysm can produce catastrophic effects undertaking both surgical and medical treatment of adenoma. Preoperative knowledge of neuroradiological findings and clinical status can help to plan the therapeutic strategy either in a single operative stage or in separate management of the two pathologies. Any single treatment is associated to significant risk of complications: aneurysm rupture in case of Cabergoline therapy as early treatment or pituitary apoplexy due to Cabergoline and double antiplatelet therapy after pseudo-aneurysm stenting. Nevertheless, as optimal planning of this conditions, securing aneurysm with flow diversion stenting permit to treat adenoma in a safer way both surgically or medically.

\section{Declaration of Interest, Funding and Acknow- ledgements}

There is no conflict of interest that could be perceived as prejudicing the impartiality of the research reported. This research did not receive any specific grant from any funding agency in the public, commercial or not-for-profit sector. Informed consent has been obtained from the patient for publication of the case report and accompanying images.

\section{References}

1. Pant B, Arita K, Kurisu K, Tominaga A, Eguchi K, et al. (1997) Incidence of intracranial aneurysm associated with pituitary adenoma. Neurosurg Rev 20: 13-17.
2. Acqui $M$, Ferrante $L$, Fraioli $B$, Cosentino $F$, Fortuna $A$, et al. (1987) Association between intracranial aneurysms and pituitary adenomas. A etiopathogenetic hypotheses. Neurochirurgia (Stuttg) 30: 177-181.

3. Hong-Seok Choi, Min-Su Kim, Young-Jin Jung, OhLyong Kim (2013) Incidental superior hypophygeal artery aneurysm embedded within pituitary adenoma. J Korean Neurosurg Soc 54: 250-252.

4. Sade B, Mohr G, Tampieri D, Rizzo A (2004) Intrasellar aneurysm and a growth hormone-secreting pituitary macroadenoma. Case report. J Neurosurg 100: 557-559.

5. Couldwell WT, Weiss MH (2004) Medical and surgical management of microprolactinoma. Pituitary 7: 31-32.

6. Amin F Saad, Almas Syed, Keyan B Marashi, Brian D O'Rourke, Joseph H Hise, et al. (2017) Endovascular therapy using flow diversion for giant internal carotid artery pseudoaneurysm arising in the setting of an invasive pituitary macroadenoma. Proc (Bayl Univ Med Cent) 30: 47-49.

7. Jakubowski J, Kendall B (1978) Coincidental aneurysms with tumours of pituitary origin. J Neurol Neurosurg Psychiatry 41: 972-979.

8. Mangiardi JR, Aleksic SN, Lifshitz M, Pinto R, Budzilovic GN, et al. (1983) Coincidental pituitary adenoma and cerebral aneurysm with pathological findings. Surg Neurol 19: 38-41.

9. Choi HS, Kim MS, Jung YJ, Kim OL (2013) Incidental superior hypophygeal artery aneurysm embedded within pituitary adenoma. J Korean Neurosurg Soc 54: 250-252.

10. Akutsu N, Hosoda K, Ohta K, Tanaka H, Taniguchi M, et al. (2014) Subarachnoid hemorrhage due to rupture of an intracavernous carotid artery aneurysm coexisting with a prolactinoma under cabergoline treatment. J Neurol Surg Rep 75: e73-e76.

11. Colao A, Di Sarno A, Landi ML, Scavuzzo F, Cappabianca $P$, et al. (2000) Macroprolactinoma shrinkage during cabergoline treatment is greater in naive patients than in patients pretreated with other dopamine agonists: A prospective study in 110 patients. J Clin Endocrinol Metab 85: 2247-2252.

12. Chng E, Dalan R (2013) Pituitary apoplexy associated with cabergoline therapy. J Clin Neurosci 20: 1637-1643.

13. Hori T, Muraoka K, Hokama Y, Takami M, Saito Y (1982) A growth-hormone-producing pituitary adenoma and an internal carotid artery aneurysm. Surg Neurol 18: 108-111.

14. Yu K, Herwadkar A, Kearney T, Gnanalingham KK (2011) Pituitary adenoma and incidental superior hypophyseal aneurysm. Br J Neurosurg 25: 432-433. 